\title{
Uncovering the role of Cow urine as bioenhancer investigated towards network pharmacology
}

\author{
komal Tilwani ${ }^{1}$ and Gayatri Dave ${ }^{1}$ \\ ${ }^{1}$ P D Patel Institute of Applied Sciences
}

October 5, 2020

\begin{abstract}
Traditional Indian medicine practice (Ayurveda) emphasized the role of "panchgavya" five products from Bos indicus for human welfare. Ayurveda classics, "Sushruta Samhita," "Ashtanga Sangraha," alluded to the therapeutic potential of pristine cow urine $(\mathrm{CU})$ as drug or drug ingredients. Compelling evidence is exhibiting the innumerable medicinal properties of CU; accordingly, this elixir can directly treat complex ailments such as leprosy, tuberculosis, and fever. Also, the classics narrated many formulations that have utilized CU for the preparation of drugs, supplemented to enhance the potency. This practice is more empirical, and only a few experimental evidence is supporting the claim are known. The associated mechanisms are poorly understood and so rendered its appeal to the limited mass. The study aims to investigate bio-enhancer like properties of CU towards network pharmacology. For that, 25 medicines having anti-bacterial, anti-fungal, anti-viral, enzyme inhibitors, and anti-inflammatory actions selected as a reference. Network analysis for twenty chemotypes found in CU was carried out. First, through enrichment analysis, the KEGG and GO terms were obtained. Second, we performed protein-protein interaction studies to screen more targets. Towards this, the drug-protein and CU- protein interactions networks built separately and processed.
\end{abstract}

\section{Graphical abstarct}




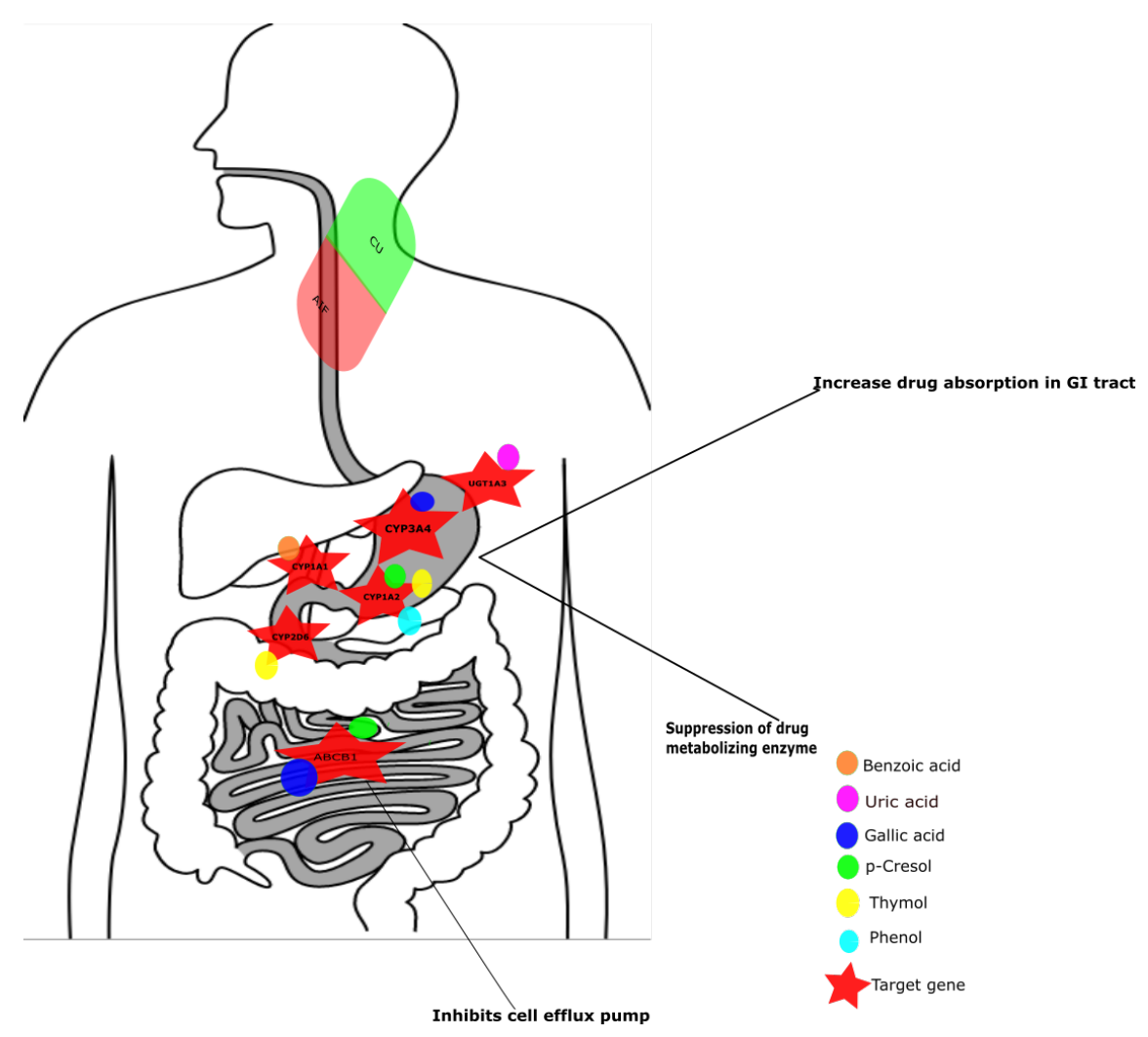

Keywords: Ayurveda; Cow-Urine; Network analysis; Molecular docking;Medicines.

\section{Introduction}

Ayurveda- an Indian traditional medicine system has adopted a holistic approach, nucleated towards balancing of five life-ruling vital factors. The repertoire includes diet, lifestyle, thought modulation, and uses of herbs $\left[{ }^{1}\right.$ Govindaraj et al.,2015; Choudhary et al.,2019]. The combinatorial practice emphasized the progressive restoration of the body through balancing these factors [Peterson et al.,2016]. In Ayurveda, a cow is bestowed; the status of the medical dispensary is a source of beaucoup products considered a boon to humanity. It provides stuff like cow dung, urine, milk, curd, and ghee (Indian butter). These altogether referred to as "panchgavya" in Ayurveda. Cow urine (CU), usually regarded as a nonessential by-product, in Ayurveda, is used in the preparation of many herbal formulations. Has medicinal value, a whole or with other active ingredients, can treat ailments, including terminal illness [Dudhatra et al.,2012]. The benefits of supplemented cow urine are thoroughly reviewed in Ayurvedic classics Charaka Samhita and Sushruta Samhita (an early journal for surgical practices). In the present era, recent studies conducted at Cow Urine Treatment and Research Centre, Indore (India), showing the positive association of cow urine (Gomutra) in treatment of disorder like blood pressure, artery blockages, and cancer [Adhikari and Joshi,2020]. First records cite its application around $4000 \mathrm{BC}$, though deep-rooted, it's compatibility towards allopathic practice needs to be established to gain broad acceptance and mass appeal. Despite extensive therapeutic uses, the method is more empirical than experimental and hence poorly recognized in a region outside the Indian subcontinent. There is a dire need for re-investigation towards established gold-standard research design of Biomedical Sciences.

Recently, United States patent [Randhawa and Sharma,2015] (No. 6,896,907 and 6,410,059) and few other laboratories are reportedly claiming for bioenhancer properties of cow urine. Generally, a bio enhancer can be natural or artificially synthesized substance/s that surges the efficacy of the drug when administered together. It increases the bioavailability of an orally administered pharmaceutical compound through several 
mechanisms. The bioenhancer often improves the solubility or adsorption of medicine, or it inhibits the action of drug-metabolizing enzymes [Javed et al.,2016] and enhances the permeability of the cytoplasmic membrane for drug entry [Peterson et al.,2019]. It's a non-exhaustive list, and a few other speculated ways of activities presented in Fig. 1.

In earlier attempts, the study of Lee et al. (2015) have noted a steep rise in the anti-inflammatory activity of apigenin when applied with resveratrol. In another study, the potency of Rifampicin greatly improved when supplemented with naturally occurring organic compound Piperidine; upon addition, the improvement in anti-tuberculosis activity was documented [Nageswari et al.,2018].

Ayurveda treatment regime advocates the application of whole cow urine or cow urine distillate. CU is a rich source of bioactive components that are known to influence multiple biological pathways towards interacting with diverse biological targets. Viewing to multi-target impact Network pharmacology is the best method to investigate as it deals with numerous networks and components in a systematic hierarchical manner [Liang et al.,2019; Zhang et al.,2017].

"Bioenhancer" -a collective term given to the compounds that enhance the potency/ efficiency or solubility of the drug when administered along-with. To elucidate the role of $\mathrm{CU}$ as a bio-enhancer using network pharmacology, we first screened 20 chemical components from cow-urine. Unique (limited to certain species) and conditions specific chemicals constituents excluded. Next, we have chosen drugs from five distinct functional categories, and such are anti-bacterial (AB), anti-fungal(AF), anti-viral, enzyme inhibitors, and anti-inflammatory (AIF). First, a network for 38 common targets of drugs (25) and CU components (20) visualized. Overlapping targets annotated through towards GO and KEGG pathway enrichment analysis. Next, towards another strategy, we built a CU-AIF core target-protein protein interaction network (PPI) and functional modules obtained. Finally, we curtailed the six key targets showing the role of CU in drug metabolism and cell signaling pathways. Molecular docking and pharmacokinetic analysis studies elucidate the inhibition of the drug-metabolizing enzyme by $\mathrm{CU}$ components. Fig. 2 represents the overall experimental approach.

\section{Experimental}

The addition of $\mathrm{CU}$ to the drug could augment the potency of the drug, to comprehend the impact, the study conducted towards taking two different approaches. First, the overlapping gene targets fetched from $\mathrm{CU}$ and medicines (from all five classes). In the second attempt, We selected only AIF drugs and conducted the PPI and topological analysis for screening of other related targets. The conventional data processing and preparation methods are typical for both approaches and narrated towards subsequent sections.

\section{Chemotype processing and target prediction}

A few reports citing the chemical nature of cow urine have been published so far, using patents, reported literature, and ayurvedic records through keyword "cow urine," names of chemical compounds were obtained. Next, sorting the chemicals to specific chemical classes, their canonical SMILES were collected from PubChem (https://pubchem.ncbi.nlm.nih.gov/) [Kim et al.,2016] and Zinc(http://zinc15.docking.org/) [Sterling and Irwin, 2015] subsequently converted to SDF formats (Table 1).Through considering the mechanism of action and commercial applications, drugs from five categories, anti-bacterial (AB), anti-fungal, anti-viral, AIF, and the enzyme inhibitors chosen to investigate the bioenhancer properties of CU.

Putative gene targets for CU components and medicines retrieved from STITCH (http://stitch.embl.de/) [Daina et al.,2016] and Swiss Target Prediction (http://www.swisstargetprediction.ch/) [Szklarczyk et al.,2019] Hits with confidence score 0.9 to 1 were considered and converted to its corresponding UniProtKB ID (https://www.uniprot.org/) for gene annotation (Supplementary Table S1). The list re-organized and manually curated using published experimental records, specifically the gene targets involved in membrane transports, such as ABC transporters and cell proton pumps, were counted (Supplementary Table S2).

\section{PPI networks and functional clusters}


Towards strategy II the network for CU and AIF PPI was visualized. A PPI network built through Bisogenet, a plug-in of Cytoscape. Bisogenet is three tire application for investigating the bimolecular relationships. It combines the information from six central PPI databases: Molecular INTeraction Database (MINT), IntAct Molecular Interaction Database (IntAct), Database of Interacting Proteins (DIP), Human Protein Reference Database (HPRD), Biomolecular Interaction Network Database (BIND), and Biological General Repository for Interaction Datasets (BioGRID) [Martin et al.,2010]. Input entities' proteins identifiers for Homo sapiens , was selected in Bisogenet, gene identifiers were uploaded, and the distance from the input node adjusted to ' 1 '. In the output column, only protein identifiers have opted. Next, through an intersection, merged PPI networks of CU-AIF targets visualized. Subsequently, we used CytoNCA, a plug-in of Cytoscape, to identify the essential proteins from the merged network. To determine the node present at a critical location, six centrality measures were selected in CytoNCA [Tang et al.,2015]. An analysis for significant centrality values such as degree centrality (DC), closeness centrality (CC), network centrality (NC), betweenness centrality (BC), local average connectivity-based method (LAC), and eigenvector centrality (EC) was conducted. Supplementary Table S3).

MCODE [Bader et al.,2003; Xiong et al.,2019], a cluster analysis algorithm in Cytoscape, detects the denselyconnected area in PPI networks. MCODE effectively builds a molecular interaction network exclusively based on connectivity (Supplementary Table S4).

\section{Functional annotation of hits of strategy I and strategy II}

In a first attempt, 38 overlapping hits uploaded to DAVID (The Database for Annotation, Visualization and Integrated Discovery; (DAVID; http://david.ncifcrf.gov; version 6.8) [Huang et al.,2007] to find out the enriched terms through Kyoto Encyclopedia of Genes and Genomes (KEGG) [Kanehisa et al.,2000] and Gene Ontology (GO) [Ashburner et al.,2000] databases. Hits with a p-value of 0.005 selected for achieving the list of significant targets. We used the E-chart gene enrichment tool for visualization of enriched GO and KEGG terms. Second, the hits of PPI clusters were analyzed, and information on associated biological pathways obtained. KEGG and GO terms from the strategy I an II screened for repetitive entries, repeated names pooled for subsequent analysis.

\section{Structure-Based Docking and activity predictions}

The overlapping GO, and KEGG terms from both the strategies were screened and further investigated for its drug binding affinities. Frail interaction of a protein-ligand was weed out through performing molecular docking in the Autodock vina ver 4.1 [Trott et al.,2010]. The X-ray crystal structures for selected targets;CYP1A1(PDB ID:6DWM), CYP1A2(PDB ID: 2HI4), UGT1A3(PDB ID: 1V4T), ABCB1(PDB ID: 6GDI), CYP3A4(PDB ID: 4I3Q), CYP2D6(PDB ID: 2F9Q), COX-1(PDB ID:6Y3C) and COX-2 (PDB ID:5KIR) were obtained from RCSB Protein Data Bank (PDB) (www. rcsb.org). Ligand- protein chemical interactions scored through visualization and interaction analysis in PyMol 1.8 [Yuan et al.,2017] and LigPlot v 4.5.3 [Laskowski and Swindells,2011]. Using PASS software, we predicted the pharmacokinetic activities for CU and drugs. Prediction of Activity Spectra for Substances (PASS) allows us to predict the mechanism of action does a ligand have on protein targets; results were re-confirmed with SwissADME (http://www.swissadme.ch/) pharmacokinetic analysis [Daina et al.,2017].

\section{Result}

\section{CU-Drugs network}

"Cow-urine can act as bioenhancer," the present study investigates the hypothesis through network pharmacology tools. Towards it, through an extensive literature review, two ligand datasets were generated. One represents CU- compounds containing 20 distinct chemotypes (dataset-1) and the other having the list of lead compounds of five commercially available drugs (dataset-2). Drugs having an analogous mechanism of action were scrutinized manually through published reports. From a class anti-viral, medicines Benazepril and Captopril inhibit the conversion of angiotensin I to angiotensin II, it shares a similar protein target, and particularly, taken to enrich the maximum genes from a biological pathways associated to the vari- 
ous medicines of the same class. Though, the selected categories of drugs are known to act on numerous macromolecules from bacteria, fungi, viruses, and Homo sapiens. Our study limits human macromolecular targets; this reduces the noise in the analysis at the end.

A ligand-target network constructed through Cytoscape (https://cytoscape.org/;version 3.8.0) [Shannon et al.,2003] shows a reasonably extensive network with 208 nodes and 234 edges (Fig. 3A). Post abandoning non-overlapping hits,38 common-hits were curtained and presented in Fig. 3B, describing 32 hub nodes and 123 edges. The hub nodes were having edges five or above were manually scrutinized and selected for further analysis.

The thirty-eight annotated targets were found associated with 76 biological processes, 25 molecular functions, 15 cellular components, and 27 KEGG pathways (Supplementary Table S5 ). The obtained results indicate that the $\mathrm{CU}$ associated targets are mainly located on the plasma membrane and involved in the metabolism and transport of small molecules, evident from gene enrichment plots. We only considered the hits with Q-value [?] 0.05 (Fig. 4A and 4B). In general, CU components enriched in following KEGG pathways: hsa00140: Steroid hormone biosynthesis, hsa00980: Metabolism of xenobiotics by cytochrome P450 hsa00982: cytochrome P450drug-metabolism pathways. The following genes, UGT1A3, CYP1A1, CYP2D6, and CYP1A2, are acting for both CU and drugs in the pathways mentioned above.

\section{PPI and clustering for AIF-CU targets}

Cellular processes are linked and accomplished by the coordinated action of functional modules. It encompasses the group of proteins involved in catering to similar functions. Towards strategy- II, the PPI networks of $\mathrm{CU}$ and AIF associated proteins developed through Bisogenet (Fig. 5A and 5B). The same nodes and edges from the two separate PPI networks were selected to obtain intersection, a merged-network containing 2016 hub nodes and 42347 edges (Fig. 5C), still complex to interpret. Post-screening through six-criterion based topological analysis in CytoNCA, 200 significant nodes were obtained (Fig. 5D). Next, the MCODE, clustering analysis gave seven significant clusters (Fig. 5E). Proteins from the modules I-V and VII are enriched in cell signaling, while the proteins from module VI are associated with the process of drug metabolisms. Overall, the results suggest that majority hits enriched to two cellular processes; cell signaling and drug metabolism (Table 2). Similar observations strike out from the analysis conducted to the strategy I; Hence a merged list of targets from both approaches curtailed contains CYP2D6, UGT1A3, CYP3A4, ABCB1, CYP1A1, and CYP1A2.

\section{Docking studies}

From a total of $4342 \mathrm{CU}$ targets, performing ligand-target docking weak interaction was weed out. Antiinflammatory drugs relieve the pain and inflammation through inhibiting the cellular cyclooxygenase (COX1 and COX2) activity, here we selected Celecoxib, diclofenac, and Aspirin as reference drugs. Post docking, the ligands were classified according to the binding affinities shown in Fig 6. The lowest binding free energy and the number of H-bonds accounted for the analysis.

The binding energy of Uric acid $(-7.2 \mathrm{kcal} / \mathrm{mol})$ is compatible with the standard drug diclofenac $(-6.4$ $\mathrm{kcal} / \mathrm{mol}$ ). For target CYP1A2, diclofenac and Uric acid use the same binding pocket evident from shared interaction with following amino acid residues; Ser279, Phe275, Gly206, His208, and Asp282 (Fig. 7A and 7B). This result also suggests that uric acid is a better candidate for the target CYP1A2 than diclofenac. For the prime drug target COX-1 the binding affinity of uric acid $(-6.6 \mathrm{kcal} / \mathrm{mol})$ is identical to diclofenac $(-6.7 \mathrm{kcal} / \mathrm{mol})$ and again a similar active site pocket where Gln144, Arg469, Lys468 are interacting with ligands and showing similar H-bond profiles (Fig. 7C and 7D). For another drug target, COX-2, the binding affinities of uric acid $(-6.9 \mathrm{kcal} / \mathrm{mol})$ are more significant than diclofenac $(-6.3 \mathrm{kcal} / \mathrm{mol})$ having the following common residues at binding pockets Gln372, Phe371, Ser121, His122 (Fig. 7E and 7F).

\section{Pharmacokinetic analysis}

Pharmacokinetic analysis of screened targets presents a promising role of cow urine in drug formulations. It suggests that CU component, gallic acid downregulates the expression of CYP3A4 and thereby delays the 
drug metabolism [Pu et al.,2015; Kollipara et al.,2014] that further increases the drug retention time in the body, eventually may result in increases in gastrointestinal absorption of a drug. Another $\mathrm{Cu}$ component, p-cresol, and Thymol inhibits the activity of CYP1A2 and CYP2D6 [Chen et al.,2013; Ekins et al.,2000] and exerts similar activity like gallic acid. p-cresol present in cow urine inhibits the membrane p-gp efflux through that it can facilitate the optimum drug delivery in the cell interior [Meesters et al.,2009; Lim et al.,2016]. Benzoic acid inhibits the activity of CYP1A1, and Uric acid regulates the activity of UGT1A3 in drug metabolism [Lu et al.,2020; Iwai et al.,2004;].

\section{Discussions}

In a grim scenario of the evolution of drug resistance and drug insensitivity, there is a need for quick and affordable solutions [Cheng et al.,2017; Kalow,1982]. The microbes acquire resistance through metabolizing the drug that poses a severe threat to existing therapeutics [Qin et al.,2019]. Discovering a novel drug entirely to combat this unforeseen change in the behavior of bacteria or cells is an economically challenging and lengthy process [Al-Baqsami et al.,2020]. The science of drug designing is perturbed by the rapid emergence of drug resistance towards established antibiotics. The discipline demands an urgent need for repositioning or reformulating the current drugs. Researcher across the globes is testing various strategies such as the inclusion of metabolic inhibitors [Twarog et al.,2020] to single- gene deletion [Elkashif et al.,2020; Lv et al.,2020] Adding bioenhancer may be one more alternative that may affect the drug metabolism [BoraSinghal et al.,2020; Tyzack and Kirchmair, 2019; Tatiraju et al.,2013] During the literature analysis for Ayurveda practice, we observed the practice of inclusion of cow urine distillate in many Ayurveda formulation, has numerous application and exert its impact through modulating the fundamental processes[Randhawa et al.,2011; Basalious et al.,2010].

Drug effectiveness depends on several biological processes, including membrane transport/binding to blood plasma proteins/vasodilation of human intestinal tight junctions/endocytosis [Randhawa,2010]. These processes mainly exalt drug absorption and distribution in the human body. The pharmacokinetics of any drug is related to many such biological processes. The results of studies conducted for the strategy I and II show that drug metabolism, one-carbon metabolism, response to the drug, cellular transports, and cell signaling pathways shared among all the selected drugs and that are also associated with CU components. Though it is in-silico studies, it firmly establishes the interaction of CU with key gene targets that often essential for drug activity. Hence, CU could be introduced as a bio enhancer. Laboratory validation studies are imperative to prove this claim further.

The elaborated results of docking and pharmacokinetic analysis display the strong association of CU with gene targets CYP2D6, UGT1A3, CYP3A4, ABCB1, CYP1A1, and CYP1A2. Typically, their gene products metabolize the drugs and facilitate their excretion for the human body.Cytochromes P450 (CYPs) are a superfamily of monooxygenase enzymes that oxidize steroids, xenobiotics, and drugs, and play a role in its clearance [Mohanty et al.,2014; Pratanwanich et al.,2014; Seden et al.,2010]. Activation or inhibition of CYP1A1, CYP2D6 CYP3A4, and CYP1A2 isoforms usually activate or prevents the detoxification of numerous xenobiotics. It is one mechanism that regulates the bioavailability of drugs. The CU components interact with these isoforms, and few are involved in its suppression, through that it reduces the rate of drug metabolism. The reduced rate of drug metabolism results in increase drug absorption and sometimes to drug toxicity.

Uridine diphosphate glucuronosyltransferase 1A3 (UGT1A3) belongs to the uridine diphosphate glucuronosyltransferase superfamily. In a mammal liver, the enzymes predominantly detoxify the endobiotics and xenobiotics and transformed them into more polar and water-soluble glucuronides [Lv et al.,2019; Jiang et al.,2015]. These altered metabolites are biologically inactive and easily extracted from the body. Drugs or herbs inhibit some members of this family may lead to the accumulation of such molecules in the body, and even sometimes, clinical toxicity observed. Though the inclusion of bio-enhancer in a drug formulation is a wise choice, it needs optimization to a certain extent; however, the in-silico studies exhibit the multilevel impact of $\mathrm{CU}$ on drug targets. 
The use of cow-urine in folklore date backs to 4000BC; however, limited knowledge of pharmacologically active compounds renders its application. It's a prima facie report that supports the ages-old Ayurveda practice that uses cow urin\{Citation\}e in preparation of many herbal formulations.

\section{References}

1. Cheng X, Lv X, Qu H, et al. Comparison of the inhibition potentials of icotinib and erlotinib against human UDP-glucuronosyltransferase 1A1.Acta Pharmaceutica Sinica B . 2017;7(6):657-664.

2. Tyzack JD, Kirchmair J. Computational methods and tools to predict cytochrome P450 metabolism for drug discovery. Chemical biology \&3 drug design . 2019;93(4):377-386.

3. Randhawa G. Cow urine distillate as bioenhancer. Journal of Ayurveda and integrative medicine . 2010;1(4):240.

4. Adhikari N, Joshi DR. Cow urine: The Total research on cow urine from the very beginning to till date.

5. Meesters RJ, Duisken M, Hollender J. Cytochrome P450-catalysed arene-epoxidation of the bioactive tea tree oil ingredient p-cymene: indication for the formation of a reactive allergenic intermediate? Xenobiotica . 2009;39(9):663-671.

6. Tang Y, Li M, Wang J, Pan Y, Wu F-X. CytoNCA: a cytoscape plugin for centrality analysis and evaluation of protein interaction networks.Biosystems . 2015;127:67-72.

7. Shannon P, Markiel A, Ozier O, et al. Cytoscape: a software environment for integrated models of biomolecular interaction networks. Genome research . 2003;13(11):2498-2504.

8. Huang DW, Sherman BT, Tan Q, et al. DAVID Bioinformatics Resources: expanded annotation database and novel algorithms to better extract biology from large gene lists. Nucleic acids research . 2007;35(suppl_2):W169-W175.

9. Mohanty I, Senapati MR, Jena D, Palai S. Diversified uses of cow urine. Int J Pharm Pharm Sci . 2014;6(3):20-2.

10. Peterson B, Weyers M, Steenekamp JH, Steyn JD, Gouws C, Hamman JH. Drug bioavailability enhancing agents of natural origin (bioenhancers) that modulate drug membrane permeation and pre-systemic metabolism.Pharmaceutics . 2019;11(1):33.

11. Nageswari AD, Rajanandh MG, Uday M, Nasreen RJ, Pujitha RR, Prathiksha G. Effect of rifampin with bio-enhancer in the treatment of newly diagnosed sputum positive pulmonary tuberculosis patients: A double-center study. Journal of clinical tuberculosis and other mycobacterial diseases . 2018;12:73-77.

12. Kalow W. Ethnic differences in drug metabolism. Clinical pharmacokinetics . 1982;7(5):373-400.

13. Ashburner M, Ball CA, Blake JA, et al. Gene ontology: tool for the unification of biology. Nature genetics . 2000;25(1):25-29.

14. Govindaraj P, Nizamuddin S, Sharath A, et al. Genome-wide analysis correlates Ayurveda Prakriti. Scientific reports . 2015;5:15786.

15. Seden K, Dickinson L, Khoo S, Back D. Grapefruit-drug interactions.Drugs . 2010;70(18):2373-2407.

16. Chen X-W, Serag ES, Sneed KB, Zhou S-F. Herbal bioactivation, molecular targets and the toxicity relevance. Chemico-biological interactions . 2011;192(3):161-176.

17. Peterson CT, Lucas J, John-Williams LS, et al. Identification of altered metabolomic profiles following a Panchakarma-based Ayurvedic intervention in healthy subjects: The Self-Directed Biological Transformation Initiative (SBTI). Scientific reports . 2016;6:32609.

18. Jiang L, Liang S-C, Wang C, et al. Identifying and applying a highly selective probe to simultaneously determine the O-glucuronidation activity of human UGT1A3 and UGT1A4. Scientific reports . 2015;5:9627.

19. Choudhary N, Singh V. Insights about multi-targeting and synergistic neuromodulators in Ayurvedic herbs against epilepsy: integrated computational studies on drug-target and protein-protein interaction networks. Scientific reports . 2019;9(1):1-23.

20. Lim HY, Ho QS, Wong KP. Interplay of metabolizing enzymes and transporter of xenobiotics. Xenobiotica . 2016;46(1):25-33. 
21. Elkashif A, Seleem MN. Investigation of auranofin and gold-containing analogues antibacterial activity against multidrug-resistant Neisseria gonorrhoeae. Scientific reports . 2020;10(1):1-9.

22. Kanehisa M, Goto S. KEGG: kyoto encyclopedia of genes and genomes.Nucleic acids research . 2000;28(1):27-30.

23. Laskowski RA, Swindells MB. LigPlot+: Multiple Ligand-Protein Interaction Diagrams for Drug Discovery. ACS Publications; 2011.

24. Kim S, Thiessen PA, Cheng T, et al. Literature information in PubChem: associations between PubChem records and scientific articles. Journal of cheminformatics . 2016;8(1):32.

25. Tatiraju DV, Bagade VB, Karambelkar PJ, Jadhav VM, Kadam V. Natural bioenhancers: An overview. $J$ Pharmacogn Phytochem . 2013;2(3):55-60.

26. Xiong Y, Yang Y, Xiong W, Yao Y, Wu H, Zhang M. Network pharmacology-based research on the active component and mechanism of the antihepatoma effect of Rubia cordifolia L. Journal of cellular biochemistry . 2019;120(8):12461-12472.

27. Lu J, Shang X, Zhong W, Xu Y, Shi R, Wang X. New insights of CYP1A in endogenous metabolism: a focus on single nucleotide polymorphisms and diseases. Acta Pharmaceutica Sinica B . 2020;10(1):91104.

28. Bora-Singhal N, Mohankumar D, Saha B, et al. Novel HDAC11 inhibitors suppress lung adenocarcinoma stem cell self-renewal and overcome drug resistance by suppressing Sox2. Scientific reports . 2020;10(1):1-20.

29. Pratanwanich N, Lió P. Pathway-based Bayesian inference of drug-disease interactions. Molecular BioSystems . 2014;10(6):1538-1548.

30. Kollipara S, Gandhi RK. Pharmacokinetic aspects and in vitro-in vivo correlation potential for lipidbased formulations. Acta Pharmaceutica Sinica B . 2014;4(5):333-349.

31. Kollipara S, Gandhi RK. Pharmacokinetic aspects and in vitro-in vivo correlation potential for lipidbased formulations. Acta Pharmaceutica Sinica B . 2014;4(5):333-349.

32. Ekins S, Ring BJ, Grace J, McRobie-Belle DJ, Wrighton SA. Present and future in vitro approaches for drug metabolism. Journal of pharmacological and toxicological methods . 2000;44(1):313-324.

33. Lv X, Xia Y, Finel M, Wu J, Ge G, Yang L. Recent progress and challenges in screening and characterization of UGT1A1 inhibitors. Acta pharmaceutica sinica b . 2019;9(2):258-278.

34. Lee J-A, Ha SK, Cho E, Choi I. Resveratrol as a bioenhancer to improve anti-inflammatory activities of apigenin. Nutrients . 2015;7(11):9650-9661.

35. Qin X, Wang X. Role of vitamin D receptor in the regulation of CYP3A gene expression. Acta Pharmaceutica Sinica B . 2019;9(6):1087-1098.

36. Iwai M, Maruo Y, Ito M, Yamamoto K, Sato H, Takeuchi Y. Six novel UDP-glucuronosyltransferase (UGT1A3) polymorphisms with varying activity. Journal of human genetics . 2004;49(3):123-128.

37. Szklarczyk D, Santos A, von Mering C, Jensen LJ, Bork P, Kuhn M. STITCH 5: augmenting proteinchemical interaction networks with tissue and affinity data. Nucleic acids research . 2016;44(D1):D380D384.

38. Daina A, Michielin O, Zoete V. SwissADME: a free web tool to evaluate pharmacokinetics, drug-likeness and medicinal chemistry friendliness of small molecules. Scientific reports . 2017;7:42717.

39. Daina A, Michielin O, Zoete V. SwissTargetPrediction: updated data and new features for efficient prediction of protein targets of small molecules. Nucleic acids research . 2019;47(W1):W357-W364.

40. Zhang R, Yu S, Bai H, Ning K. TCM-Mesh: The database and analytical system for network pharmacology analysis for TCM preparations.Scientific reports . 2017;7(1):1-14.

41. Javed S, Ahsan W, Kohli K. The concept of bioenhancers in bioavailability enhancement of drugs-a patent review. J Sci Lett . 2016;1:143-65.

42. Pu Q-H, Shi L, Yu C. Time-dependent inhibition of CYP3A4 by gallic acid in human liver microsomes and recombinant systems. Xenobiotica . 2015;45(3):213-217.

43. Yuan S, Chan HS, Hu Z. Using PyMOL as a platform for computational drug design. Wiley Interdisciplinary Reviews: Computational Molecular Science . 2017;7(2):e1298.

44. Lv C, Huang L. Xenobiotic receptors in mediating the effect of sepsis on drug metabolism. Acta 
Pharmaceutica Sinica B . 2020;10(1):33-41.

45. Sterling T, Irwin JJ. ZINC 15-ligand discovery for everyone.Journal of chemical information and modeling . 2015;55(11):2324-2337.

46. Al-Baqsami ZF, Ahmad S, Khan Z. Antifungal drug susceptibility, molecular basis of resistance to echinocandins and molecular epidemiology of fluconazole resistance among clinical Candida glabrata isolates in Kuwait. Scientific reports . 2020;10(1):1-10.

47. Bader GD, Hogue CW. An automated method for finding molecular complexes in large protein interaction networks. BMC bioinformatics . 2003;4(1):2.

48. Bader GD, Hogue CW. An automated method for finding molecular complexes in large protein interaction networks. BMC bioinformatics . 2003;4(1):2.

49. Dudhatra GB, Mody SK, Awale MM, et al. A comprehensive review on pharmacotherapeutics of herbal bioenhancers. The Scientific World Journal . 2012;2012.

50. Martin A, Ochagavia ME, Rabasa LC, Miranda J, Fernandez-de-Cossio J, Bringas R. BisoGenet: a new tool for gene network building, visualization and analysis. BMC bioinformatics . 2010;11(1):91.

51. Randhawa GK, Jagdev Singh Kullar R. Bioenhancers from mother nature and their applicability in modern medicine. International journal of applied and basic medical research . 2011;1(1):5.

52. Randhawa GK, Sharma R. Chemotherapeutic potential of cow urine: A review. Journal of intercultural ethnopharmacology . 2015;4(2):180.

53. Trott O, Olson AJ. AutoDock Vina: improving the speed and accuracy of docking with a new scoring function, efficient optimization, and multithreading. Journal of computational chemistry . 2010;31(2):455-461.

54. Twarog NR, Connelly M, Shelat AA. A critical evaluation of methods to interpret drug combinations. Scientific reports . 2020;10(1):1-13.

Table

\section{BENZOIDS}

Benzoic acid

p-Cresol

Phenol

Gallic acid

Salicylic acid

ORGANIC OXYGEN COMPOUND

Lactose

ORGANIC ACID AND DERIVATIVES

Creatinine

LIPID AND LIPID-LIKE MOLECULE

Thymol
HOMOGENEOUS NON-METAL COMPOUND

Chloride

Copper

Phosphate

Sulfate

Nitrite

Phosphorus Pentoxide

ORGANO HETEROCYCLIC COMPOUND

Uric acid

Allantoin

Nicotine

PHENYLPROPENOIDS AND POLYKETIDES

Ferulic acid

Caffeic acid

Table 1. Chemotypes are commonly present in cow urine, a class-wise summary.

\begin{tabular}{lll}
\hline Cluster classification & & Gene targets \\
\hline I Signaling pathways & Q-value & Associated protein targets \\
hsa04020: Calcium signaling pathway & $2.00 \times 10^{-03}$ & GNA15, LTB4R2, ADRA1A \\
hsa04080: Neuroactive ligand-receptor interaction & $4.70 \times 10^{-03}$ & P2RY2, LTB4R2, ADRA1A \\
hsa04062: Chemokine signaling pathway & $2.10 \times 10^{-03}$ & CCL13, CXCL5, CXCL6 \\
hsa04060: Cytokine-cytokine receptor interaction & $3.60 \times 10^{-03}$ & CCL13, CXCL5, CXCL6 \\
hsa04010: MAPK signaling pathway & $4.90 \times 10^{-05}$ & PTPN7, DUSP4, DUSP2, DUSP6 \\
hsa00140: Steroid hormone biosynthesis & $1.70 \times 10^{-02}$ & UGT1A3, AKR1C1
\end{tabular}


II Metabolism

hsa00980: Metabolism of xenobiotics by cytochrome P450

$1.10 \times 10^{-04}$

UGT1A3, CYP2D6, AKR1C1

hsa00982: Drug metabolism - cytochrome P450

$2.00 \times 10^{-03} \quad$ UGT1A3, CYP2D6

Table 2. List of significant pathways and genes obtained through clustering and enrichment analysis.

\section{Figure legends}

Figure 1. Possible ways towards which bio-enhancer can enhance drug potency.

Figure 2. Experimental strategy.

Figure 3A. Compound- drug targets network.

Figure 3B. CU-Drugs shared target network, sorted to degree centrality (red color) and confidence score, shows ranked order for 32 hub nodes.

Figure 4: Functional enrichment analysis of the 32 overlapping hits. (A) it represents a graded list of enriched pathways. (B) enriched GO terms (biological processes). The color scale correlates to the significant Q-values, and the sizes of the dots represent the number of genes corresponding to each node.

Figure 5. CU-AIF PPI network. (A) CU-related targets PPI network (B) AIF-related targets PPI network. (C) Intersection of PPI networks. (D) PPI network by the screening criteria of 'DC [?] 4.0' 'EC' [?]10, 'LAC' [?] 4.0, 'BC' [?] 100 , 'CC' [?]4 and 'NC' [?]5.0'. (E) Clusters of core-target PPI network.

Figure 6. Heat map for docking score. The depth of color represents the docking score; the higher the absolute value of the score, the strong the binding.

Figure 7. Molecular docking analysis; (A ) Receptor-ligand interaction binding pose of uric acid against CYP1A2. (B) diclofenac- CYP1A2 (C) uric acid- COX-1. (D) diclofenac - COX-1.(E) uric acid - COX-2.(F) diclofenac-COX-2.

\section{Figures}




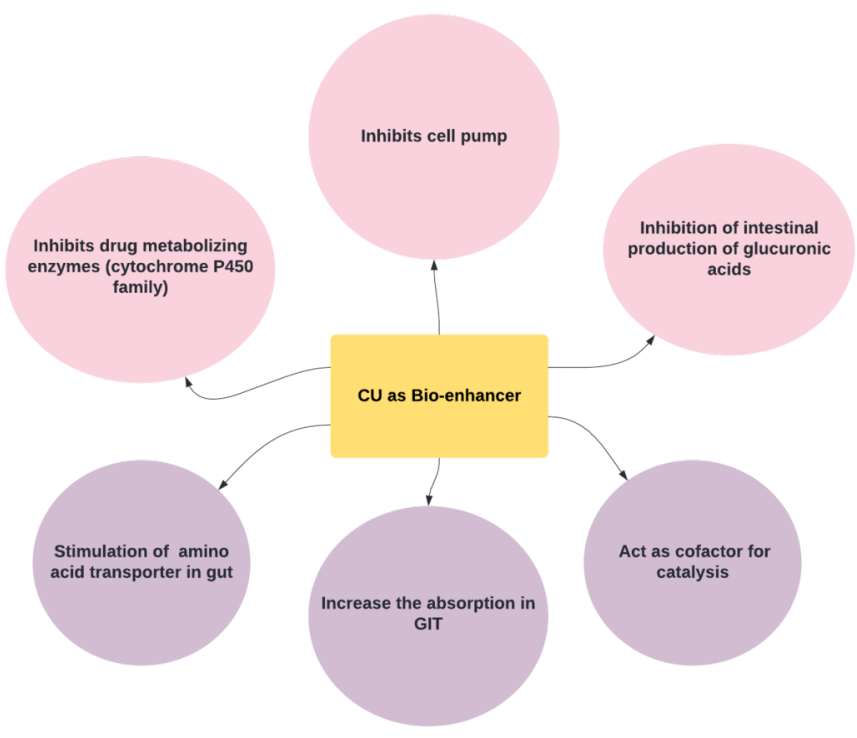

Figure 1

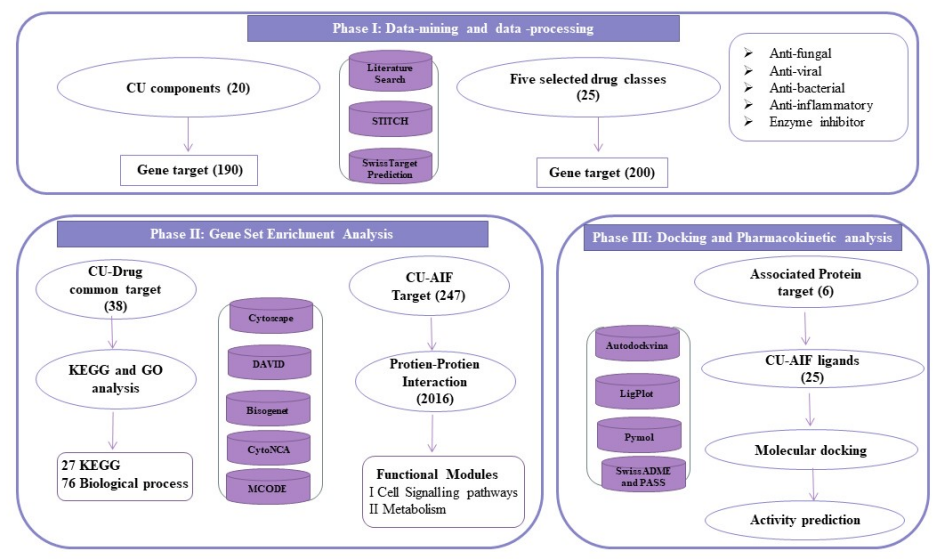

Figure 2 


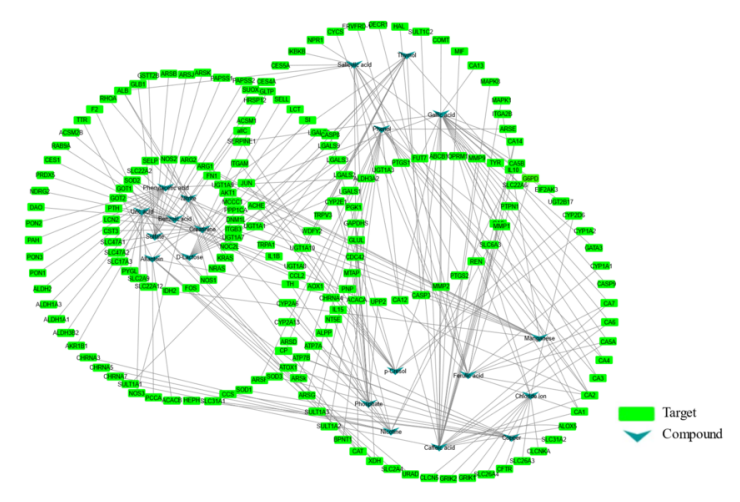

Figure 3A

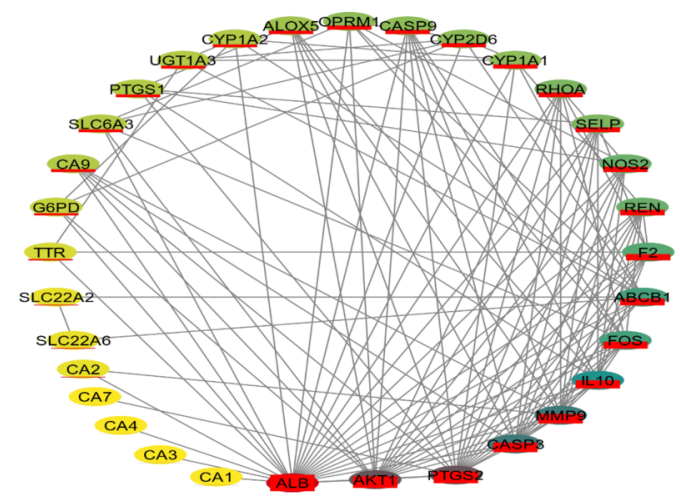

Figure 3B 


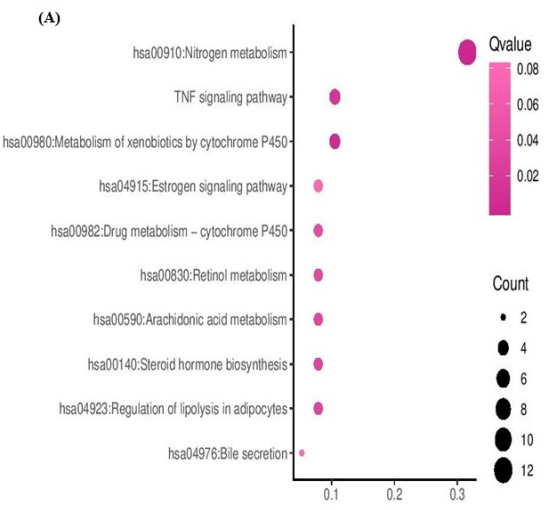

Figure 4A

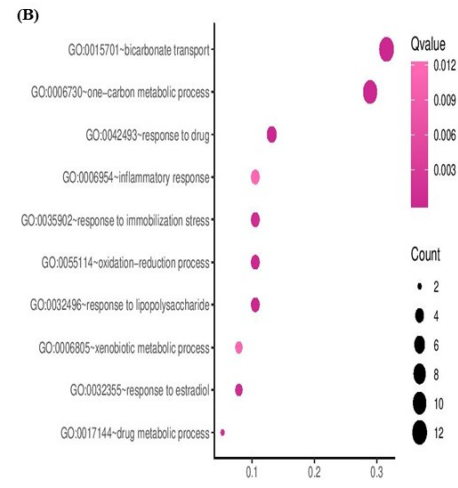

Figure 4B 


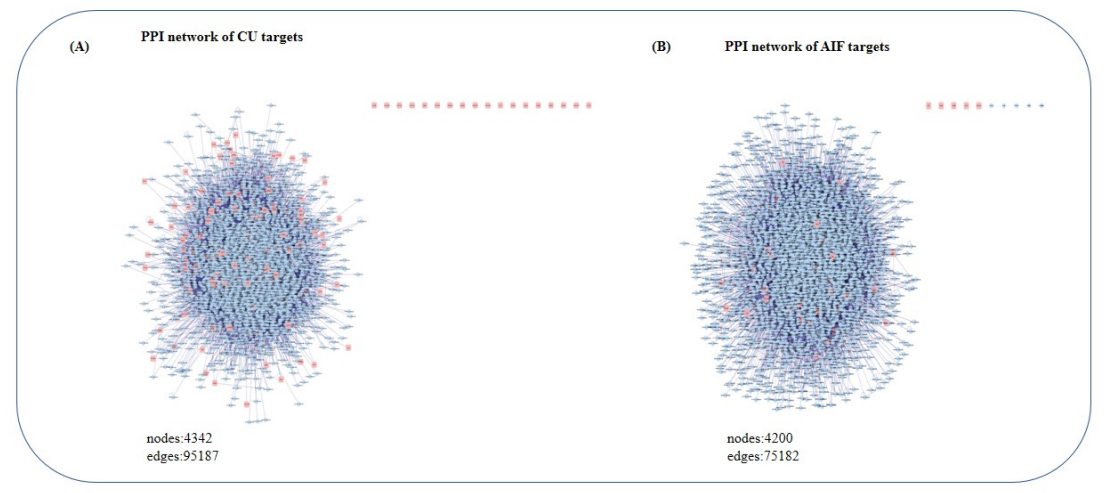

Figure $5 \mathrm{~A}$ and $5 \mathrm{~B}$

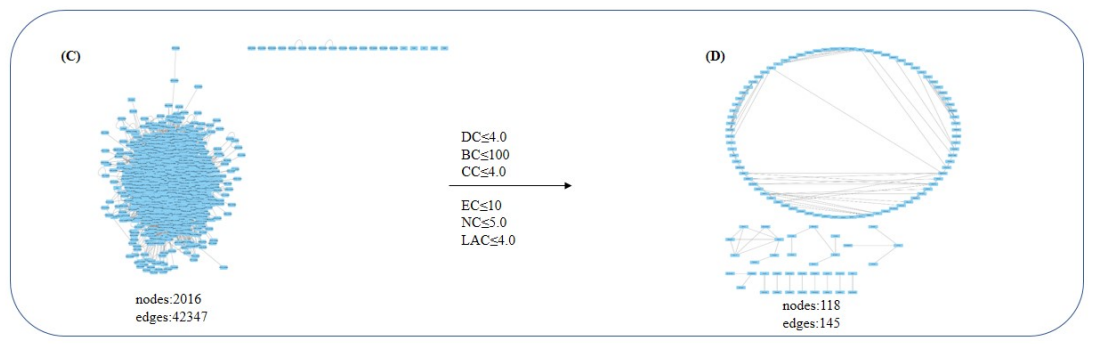

Figure 5Cand 5D 


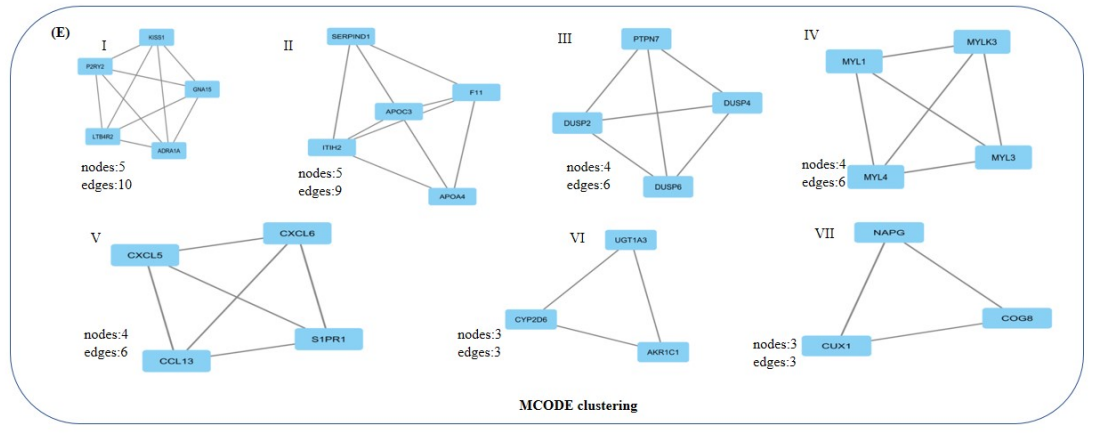

Figure 5E

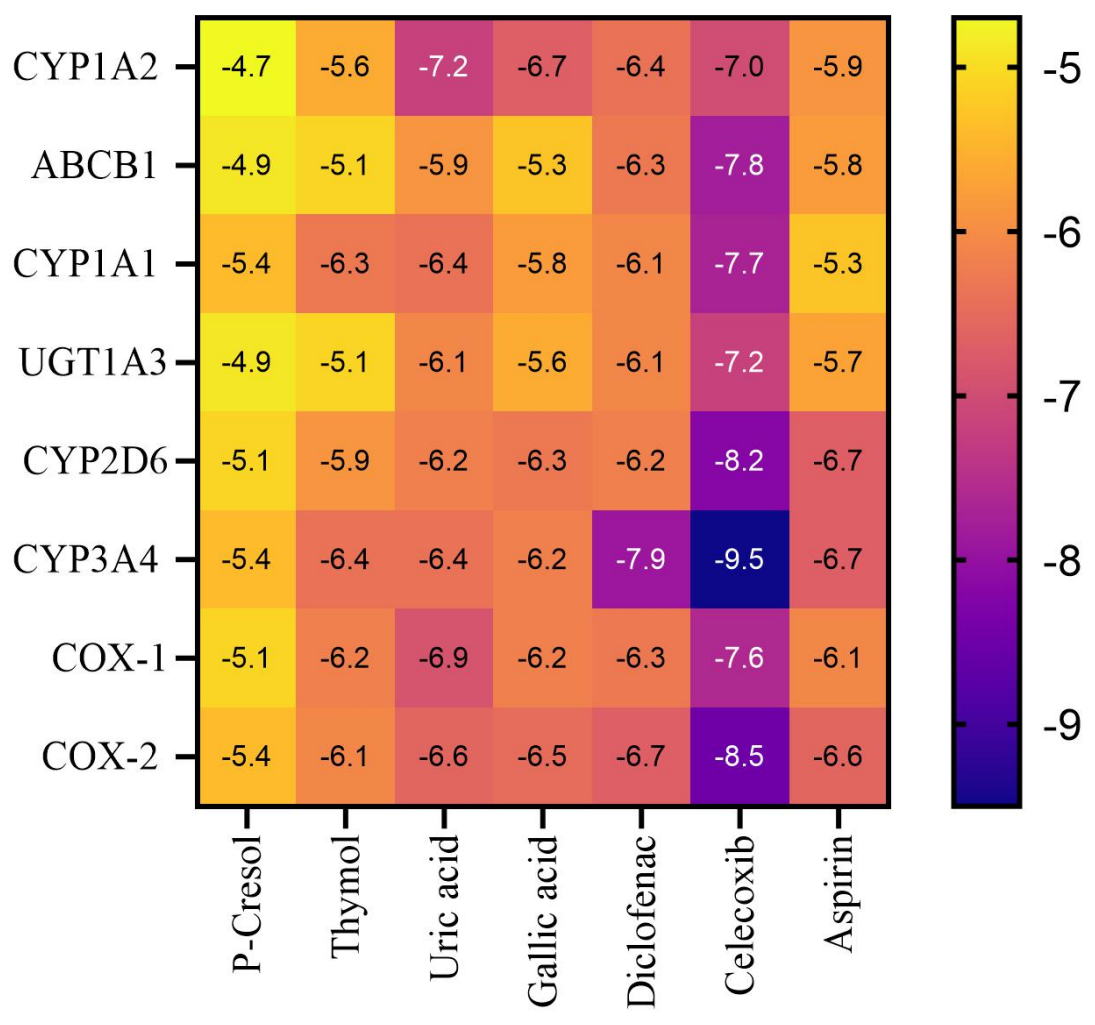

Figure 6 

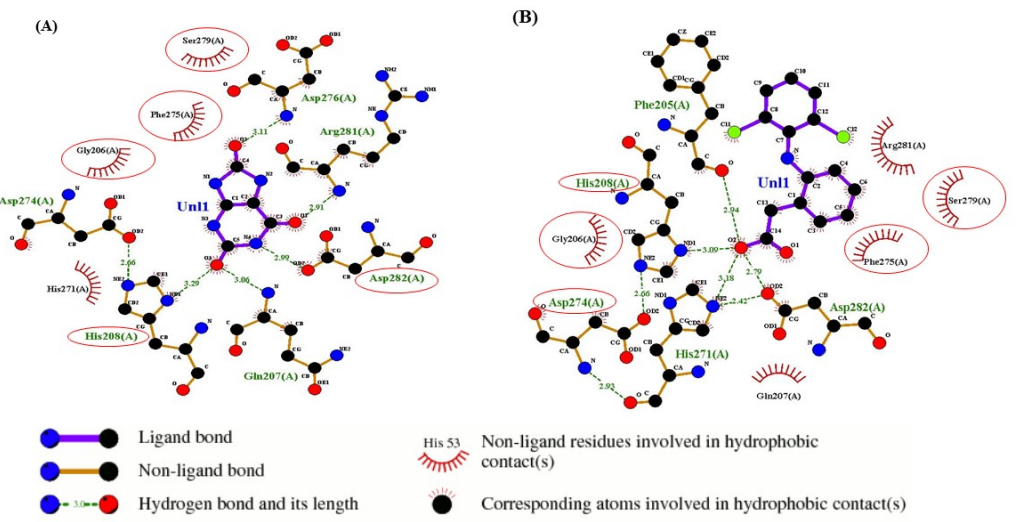

Figure $7 \mathrm{~A}$ and $7 \mathrm{~B}$

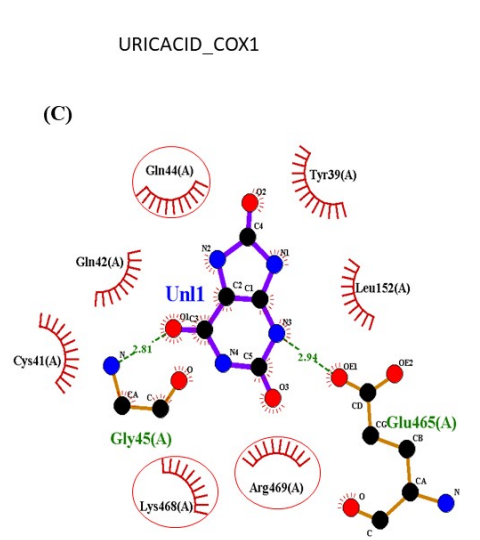

DICLOFENAC_COX1

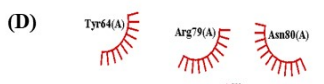

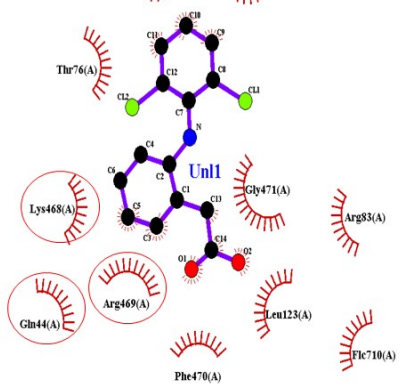

Figure $7 \mathrm{C}$ and $7 \mathrm{D}$ 
a. Uricacid COX2

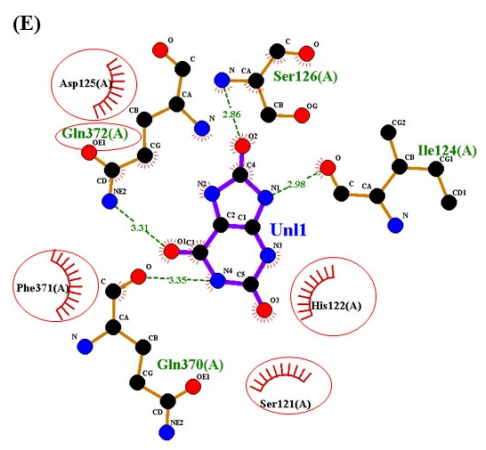

DICLOFENAC

(F)

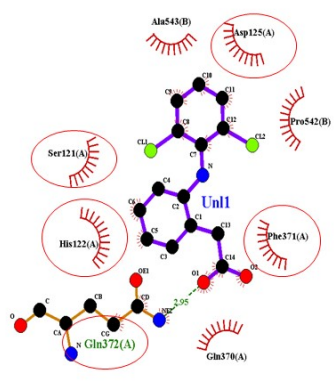

Figure $7 \mathrm{E}$ and $7 \mathrm{~F}$ 